\title{
The density Turán problem for 3-uniform linear hypertrees. An efficient testing algorithm
}

\begin{abstract}
Let $\mathcal{T}=(V, \mathcal{E})$ be a 3-uniform linear hypertree. We consider a blow-up hypergraph $\mathcal{B}[\mathcal{T}]$. We are interested in the following problem. We have to decide whether there exists a blow-up hypergraph $\mathcal{B}[\mathcal{T}]$ of the hypertree $\mathcal{T}$, with hyperedge densities satisfying some conditions, such that the hypertree $\mathcal{T}$ does not appear in a blow-up hypergraph as a transversal. We present an efficient algorithm to decide whether a given set of hyperedge densities ensures the existence of a 3 -uniform linear hypertree $\mathcal{T}$ in a blow-up hypergraph $\mathcal{B}[\mathcal{T}]$.
\end{abstract}

1. Introduction. Let $\mathcal{H}=(V, \mathcal{E})$ be a simple, connected and finite hypergraph with the vertex set $V$ and hyperedge set $\mathcal{E}$ (see [2]). Turán [12] stated the first results in extremal graph theory. Then many authors extended this subject and formulated similar and new Turán density problems. In $[1,5,6,8,10,11]$ and [13] interesting results for some families of graphs are obtained.

In this paper we present an algorithm for testing whether a hypertree with a given set of hyperedge densities is a factor (transversal) of a blow-up hypergraph. Our algorithm has the time complexity at most $\mathcal{O}\left(n^{2}\right)$, where $n$ is the number of hyperedges of the hypertree.

2010 Mathematics Subject Classification. 05C32, 05C42.

Key words and phrases. Uniform linear hypertree, blow-up hypergraph, transversal, Turán density. 
Csikvári and Nagy [7] discovered some interesting algorithm for testing whether a tree with a given set of edge densities is a factor of a blowup graph. We extend their algorithm to the family of 3-uniform linear hypertrees.

Now we define some notions and notations. Other definitions one can find in $[2,4]$ and [9]. A hypergraph $\mathcal{H}$ is called linear if any two hyperedges intersect in at most one vertex. A hypergraph $\mathcal{H}$ is called $r$-uniform if each hyperedge consists of $r$ vertices. A subhypergraph $\mathcal{P}_{t}$ of $\mathcal{H}$ is called a linear hyperpath of length $t$ if the hyperedges of $\mathcal{P}_{t}$ can be labelled by $e_{i}, 0 \leq i \leq t-1$ such that the sequence $\left(e_{0}, e_{1}, e_{2}, \ldots, e_{t-1}\right)$ satisfies the condition: $\left|e_{i} \cap e_{j}\right|=1$ if and only if $|i-j|=1$ and $e_{i} \cap e_{j}=\emptyset$ if and only if $|i-j|>1$, where $e_{i} \in \mathcal{E}(\mathcal{H})$ (see Figure 1(a)). A subhypergraph $\mathcal{C}_{t}$ of $\mathcal{H}, t \geq 3$, is called a linear hypercycle of length $t$ if the hyperedges of $\mathcal{C}_{t}$ can be labelled by $e_{i}, 0 \leq i \leq t-1$ such that the sequence $\left(e_{0}, e_{1}, e_{2}, . ., e_{t-1}\right)$ satisfies the condition: $\left|e_{i} \cap e_{j}\right|=1$ if and only if $|i-j|=1$ or $i=0$ and $j=t-1$ and $e_{i} \cap e_{j}=\emptyset, i \neq j$, in the opposite case, where $e_{i} \in \mathcal{E}(\mathcal{H})$. Differences of indices are taken $\bmod (t-1)$ (see Figure 2). An $r$-uniform linear hypertree $\mathcal{T}$ is a connected linear $r$-uniform hypergraph without linear hypercycles (see Figure 3).
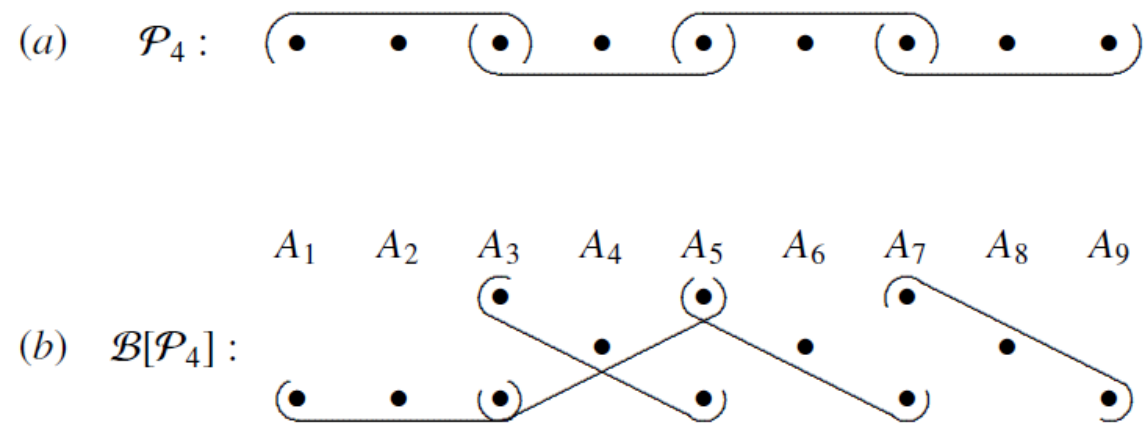

FiguRE 1. A 3-uniform linear hyperpath on 4 hyperedges and a blow-up hypergraph $\mathcal{B}\left[\mathcal{P}_{4}\right]$ without a factor $\mathcal{P}_{4}$. Let $\left|A_{1}\right|=\left|A_{2}\right|=\left|A_{4}\right|=\left|A_{6}\right|=\left|A_{8}\right|=\left|A_{9}\right|=1$ and $\left|A_{3}\right|=\left|A_{5}\right|=\left|A_{7}\right|=2$. We obtain the following densities between the clusters in $\mathcal{B}\left[\mathcal{P}_{4}\right]: d\left(A_{1}, A_{2}, A_{3}\right)=d\left(A_{3}, A_{4}, A_{5}\right)=$ $d\left(A_{7}, A_{8}, A_{9}\right)=\frac{1}{2}, d\left(A_{5}, A_{6}, A_{7}\right)=\frac{1}{4}$ and 0 for others. If we add the new hyperedge between clusters $A_{7}, A_{8}, A_{9}$, we get $d\left(A_{7}, A_{8}, A_{9}\right)=1$ and $\mathcal{P}_{4}$ as a factor.

A set $S \subset V(\mathcal{H})$ is called an independent vertex set if the subhypergraph of $\mathcal{H}$ induced by $S$ has an empty set of hyperedges. The degree of a vertex $v$ in the hypergraph $\mathcal{H}$ is the number of hyperedges containing this vertex. 


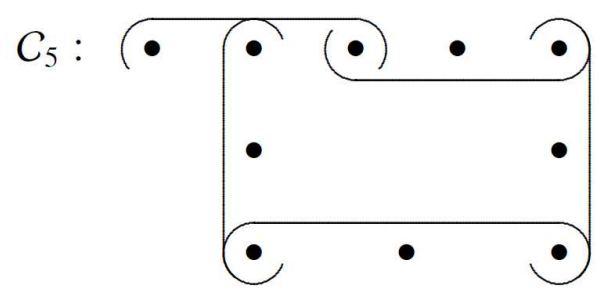

Figure 2. A 3-uniform linear hypercycle on 5 hyperedges.

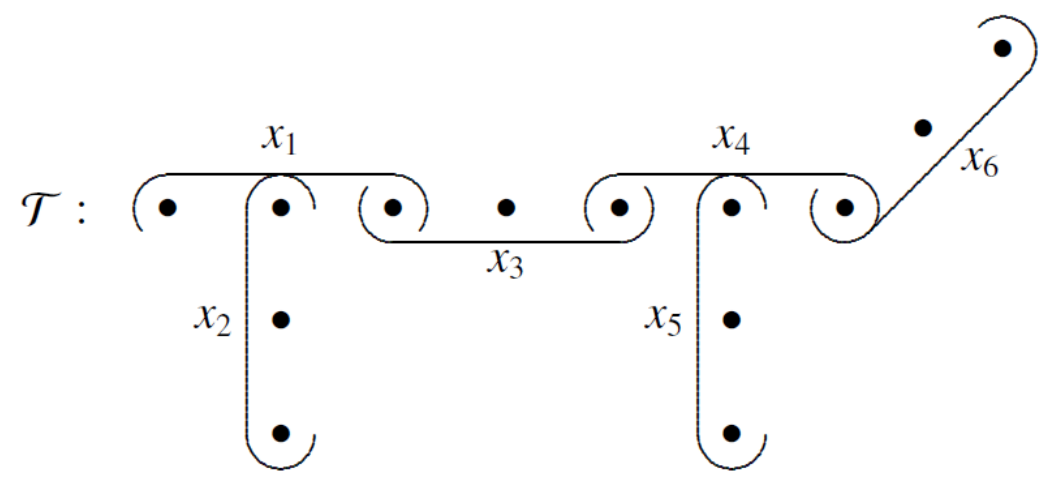

FiguRE 3. A 3-uniform linear hypertree $\mathcal{T}=(V, \mathcal{E})$, where $|V(\mathcal{T})|=13$ and $|\mathcal{E}(\mathcal{T})|=6$, with variables $x_{e}$ assigned to hyperedges.

Each vertex of degree 1 in a hypergraph $\mathcal{H}$ is called a leaf. We say that the hypergraph $\mathcal{H}$ is $r$-regular if each vertex of $\mathcal{H}$ has degree $r$. A hyperedge $e \in \mathcal{E}(\mathcal{H})$ is called a pendant hyperedge if it contains exactly one vertex of degree $>1$. The set $M \subseteq \mathcal{E}(\mathcal{H})$ is called a matching (or independent hyperedge set) in the hypergraph $\mathcal{H}$ if the subhypergraph of $\mathcal{H}$ induced by $M$ is 1-regular. Let $\mathcal{H}$ be a 3 -uniform linear hypergraph. For each vertex $i \in V(\mathcal{H})$ we associate a cluster $A_{i}$, as a set of new, independent vertices. For a hypergraph $\mathcal{H}$ we define a blow-up hypergraph $\mathcal{B}[\mathcal{H}]$ of the hypergraph $\mathcal{H}$ as follows. First we replace each vertex $i \in V(\mathcal{H})$ by a cluster $A_{i}$ and next we create some hyperedges between the clusters $A_{i}, A_{j}$ and $A_{k}$ if $\{i, j, k\}$ is a hyperedge in $\mathcal{H}, i, j, k \in V(\mathcal{H})$. Equivalently each hyperedge in $\mathcal{B}[\mathcal{H}]$ has exactly one vertex from the clusters.

For any three clusters we define a density between them by the following formula

$$
d\left(A_{i}, A_{j}, A_{k}\right)=\frac{e\left(A_{i}, A_{j}, A_{k}\right)}{\left|A_{i}\right|\left|A_{j}\right|\left|A_{k}\right|}
$$


where $e\left(A_{i}, A_{j}, A_{k}\right)$ denotes the number of hyperedges with one element of each of the clusters $A_{i}, A_{j}$ and $A_{k}$.

The hypergraph $\mathcal{H}$ is a transversal of $\mathcal{B}[\mathcal{H}]$ if $\mathcal{H}$ is a subhypergraph of $\mathcal{B}[\mathcal{H}]$ such that we have a homomorphism

$$
\phi: V(\mathcal{H}) \rightarrow V(\mathcal{B}[\mathcal{H}])
$$

for which $\phi(i) \in A_{i}$ for all $i \in V(\mathcal{H})$. Other terminology: $\mathcal{H}$ is a factor of $\mathcal{B}[\mathcal{H}]$ (see Figure $1(\mathrm{~b})$ ).

A hyperedge $e=\{i, j, k\}$ of the hypergraph $\mathcal{H}$ we denote shortly by $e=i j k$.

The homogeneous density Turán problem for 3-uniform linear hypergraphs can be defined as follows. Let us determine the critical hyperedge density, denoted by $d_{c r i t}(\mathcal{H})$, which ensures the existence of the subhypergraph $\mathcal{H}$ of $\mathcal{B}[\mathcal{H}]$ as a transversal. Precisely, assume that all hyperedges $e=$ $\{i, j, k\}$ in the hypergraph $\mathcal{H}$ satisfy the condition $d\left(A_{i}, A_{j}, A_{k}\right)>d_{\text {crit }}(\mathcal{H})$, where $i, j, k \in V(\mathcal{H})$. Then, no matter how we construct the blow-up hypergraph $\mathcal{B}[\mathcal{H}]$, it contains the hypergraph $\mathcal{H}$ as a transversal. In other words, for any value $d<d_{\text {crit }}(\mathcal{H})$ there exists a blow-up hypergraph $\mathcal{B}[\mathcal{H}]$ which does not contain $\mathcal{H}$ as a transversal and such that $d\left(A_{i}, A_{j}, A_{k}\right)>d$ for all hyperedges $i j k \in \mathcal{E}(\mathcal{H})$.

Moreover, we define the inhomogeneous density Turán problem for 3uniform linear hypergraphs as follows. Let us assume that for every hyperedge $e \in \mathcal{E}(\mathcal{H})$ a density $\gamma_{e}$ is given. Now our task is to decide if the set of densities $\left\{\gamma_{e}\right\}_{e \in \mathcal{E}(\mathcal{H})}$ ensures the existence of the hypergraph $\mathcal{H}$ as a transversal or we can construct a blow-up hypergraph $\mathcal{B}[\mathcal{H}]$ such that $d\left(A_{i}, A_{j}, A_{k}\right) \geq \gamma_{i j k},\{i, j, k\} \in \mathcal{E}(\mathcal{H})$, but it does not induce the hypergraph $\mathcal{H}$ as a transversal.

These two problems have been studied in $[7,11]$ for simple graphs which are 2-uniform linear hypergraphs. We extend some of these results to 3 -uniform linear hypertrees.

Let us recall the definition of the multivariate matching polynomial of the hypergraph.

Let $\mathcal{H}$ be a hypergraph and let $x_{e}$ be the vector of variables $x_{e}$, for $e \in \mathcal{E}(\mathcal{H})$. We define the multivariate matching polynomial $F_{\mathcal{H}}\left(x_{e}, t\right)$ of the hypergraph $\mathcal{H}$ as follows

$$
F_{\mathcal{H}}\left(\underline{x_{e}}, t\right)=\sum_{M \in \mathcal{M}}\left(\prod_{e \in M} x_{e}\right)(-t)^{|M|},
$$

where the summation goes over all matchings of the hypergraph $\mathcal{H}$, including the empty matching (see Example 1 below).

The polynomial is a useful tool for the proofs of our results. 
Example 1. Let us consider the 3-uniform linear hypertree $\mathcal{T}$ with 6 hyperedges as in Figure 3. Assume that variables $x_{e}$ are given for hyperedges $e \in \mathcal{E}(\mathcal{T})$ as follows

$$
x_{1}=2, x_{2}=1, x_{3}=3, x_{4}=2, x_{5}=3, x_{6}=1 .
$$

Then the multivariate matching polynomial of the hypertree $\mathcal{T}$ is presented below

$$
\begin{aligned}
F_{\mathcal{T}}\left(\underline{x_{e}}, t\right)= & 1-t\left(x_{1}+x_{2}+x_{3}+x_{4}+x_{5}+x_{6}\right) \\
& +t^{2}\left(x_{1} x_{4}+x_{1} x_{5}+x_{1} x_{6}+x_{2} x_{3}+x_{2} x_{4}\right. \\
& \left.+x_{2} x_{5}+x_{2} x_{6}+x_{3} x_{5}+x_{3} x_{6}+x_{5} x_{6}\right) \\
& -t^{3}\left(x_{1} x_{5} x_{6}+x_{2} x_{3} x_{5}+x_{2} x_{3} x_{6}+x_{2} x_{5} x_{6}+x_{3} x_{5} x_{6}\right) \\
& +t^{4} x_{2} x_{3} x_{5} x_{6}=1-12 t+36 t^{2}-30 t^{3}+9 t^{4} .
\end{aligned}
$$

\section{The inhomogeneous density Turán problem for 3-uniform linear} hypertrees. In this section we study the inhomogeneous density Turán problem for 3-uniform linear hypertrees $\mathcal{T}$, where a hyperedge density $\gamma_{e}$ is given for each hyperedge $e \in \mathcal{E}(\mathcal{T})$. We extend some results presented in [7], where the authors studied the inhomogeneous problem for trees and proved the following theorem.

Theorem 1 (Csikvári, Nagy [7]). Let $T$ be a tree of order $n$ and let $v$ be a leaf of $T$. Assume that for each edge of $T$ a density $\gamma_{e}=1-r_{e}$ is given. Let $T^{\prime}$ be a tree obtained from $T$ by deleting the leaf $v$ and the edge uv, where $u$ is the unique neighbour of $v$. Let the edge densities $\gamma_{e}^{\prime}$ in $T^{\prime}$ be defined as follows

$$
\gamma_{e}^{\prime}= \begin{cases}\gamma_{e}=1-r_{e}, & \text { if } e \text { is not incident to } u \\ 1-\frac{r_{e}}{1-r_{u v}}, & \text { if } e \text { is incident to } u\end{cases}
$$

Then the set of densities $\left\{\gamma_{e}\right\}_{e \in E(T)}$ ensures the existence of the factor $T$ if and only if all $\gamma_{e}^{\prime} \in(0,1]$ and the set of densities $\left\{\gamma_{e}^{\prime}\right\}_{e \in E\left(T^{\prime}\right)}$ ensures the existence of the factor $T^{\prime}$.

Theorem 1 provides the authors of [7] with an efficient algorithm to decide whether a given set of edge densities in a tree ensures the existence of a transversal or does not ensure. We show that their algorithm can be extended for 3-uniform linear hypertrees. This extension is presented in Algorithm $\mathcal{T}$. 


\section{Algorithm $\mathcal{T}$ (for 3-uniform linear hypertrees)}

Input: a 3-uniform linear hypertree $\mathcal{T}$ with the set of hyperedge densities $\left\{\gamma_{e}\right\}_{e \in \mathcal{E}(\mathcal{T})}$.

Output: a boolean value $D=\left\{\begin{array}{l}\text { TRUE, the densities } \gamma_{e} \text { ensure the existence of a factor } \mathcal{T}, \\ F A L S E, \text { the densities } \gamma_{e} \text { does not ensure the existence of a factor } \mathcal{T} .\end{array}\right.$

Consider a weighted hypertree $\left(\mathcal{T}, \underline{r_{e}}\right)$, where $r_{e}=1-\gamma_{e}$.

Step 0 . IF $|V(\mathcal{T})| \geq 3$ and there exists a hyperedge $e \in \mathcal{E}(\mathcal{T})$ for which $r_{e} \geq 1$

THEN $D:=F A L S E ;$ STOP;

Step 1. IF $|V(\mathcal{T})|=3$ (means $\mathcal{T}$ is a hyperpath $\mathcal{P}_{1}$ ) and $0 \leq r_{e}<1$

THEN $D:=$ TRUE; STOP;

Step 2. IF $|V(\mathcal{T})|>3$

THEN pick two leaves $u, v$ from a pendant hyperedge $f=\{u, v, w\} \in \mathcal{E}(\mathcal{T})$.

Let $\mathcal{T}^{\prime}=(V(\mathcal{T})-\{u, v\}, \mathcal{E}(\mathcal{T})-\{u, v, w\})$ and for each hyperedge $e \in \mathcal{E}\left(\mathcal{T}^{\prime}\right)$ set

$$
r_{e}^{\prime}= \begin{cases}r_{e}, & \text { if } e \cap f=\emptyset, \\ \frac{r_{e}}{1-r_{u v w}}, & \text { if } e \cap f=\{w\}\end{cases}
$$

STOP;

IF $r_{e}^{\prime} \geq 1$ for some hyperedge $e \in \mathcal{E}\left(\mathcal{T}^{\prime}\right)$ THEN $D:=F A L S E$;

GO TO Step 1 with $\left(\mathcal{T}, \underline{r_{e}}\right):=\left(\mathcal{T}^{\prime}, r_{e}^{\prime}\right)$.

Proposition 1. Algorithm $\mathcal{T}$ stops in at most $\mathcal{O}\left(n^{2}\right)$ steps, where $n$ is the number of hyperedges of the input hypertree.

The correctness of Algorithm $\mathcal{T}$ follows from the following theorem.

Theorem 2. Let $\mathcal{T}=(V, \mathcal{E})$ be a 3-uniform linear hypertree, where $|\mathcal{E}(\mathcal{T})| \geq$ 2. Let $u, v$ be two leaves of $\mathcal{T}$ which lie in the hyperedge $e=\{u, v, w\} \in \mathcal{E}(\mathcal{T})$ for some $w \in V(\mathcal{T})$. Assume that for each hyperedge of $\mathcal{T}$ the density $\gamma_{e}=1-r_{e}$ is given. Let $\mathcal{T}^{\prime}$ be a hypertree obtained from $\mathcal{T}$ by deleting 
leaves $u$ and $v$ with the hyperedge uvw. Let the hyperedge densities $\gamma_{e}^{\prime}$ in $\mathcal{T}^{\prime}$ be defined as follows

$$
\gamma_{e}^{\prime}= \begin{cases}\gamma_{e}=1-r_{e}, & \text { if } e \text { is not incident to } w \text { in } \mathcal{T}^{\prime} \\ 1-\frac{r_{e}}{1-r_{u v w}}, & \text { if } e \text { is incident to } w \text { in } \mathcal{T}^{\prime} .\end{cases}
$$

Then the set of densities $\left\{\gamma_{e}\right\}_{e \in \mathcal{E}(\mathcal{T})}$ ensures the existence of a factor $\mathcal{T}$ if and only if all $\gamma_{e}^{\prime} \in(0,1]$ and the set of densities $\left\{\gamma_{e}^{\prime}\right\}_{e \in \mathcal{E}\left(\mathcal{T}^{\prime}\right)}$ ensures the existence of a factor $\mathcal{T}^{\prime}$.

Proof. Let $\mathcal{T}$ be a 3-uniform linear hypertree and let a density $\gamma_{e}=1-r_{e}$ be given for each $e \in \mathcal{E}(\mathcal{T}),|\mathcal{E}(\mathcal{T})| \geq 2$.

$(\Leftarrow)$ First we prove the following statement: if all $\gamma_{e}^{\prime}$ are indeed densities and they ensure the existence of a factor $\mathcal{T}^{\prime}$, then the original densities $\gamma_{e}$ ensure the existence of a factor $\mathcal{T}$.

Let $\mathcal{B}[\mathcal{T}]$ be a blow-up hypergraph of the hypertree $\mathcal{T}$ such that the density between clusters $A_{i}, A_{j}$ and $A_{k}$ is at least $\gamma_{i j k}$, where $A_{i}, A_{j}, A_{k}$ are clusters of the vertices and $i, j, k \in V(\mathcal{T})$. We show that $\mathcal{B}[\mathcal{T}]$ contains a factor $\mathcal{T}$.

Let $u, v, w \in V(\mathcal{T})$ and $\{u, v, w\} \in \mathcal{E}(\mathcal{T})$, where $u, v$ are leaves. Define $R_{u, v, w}$ as a subset of $A_{w}$ in the following way (see Figure 4)

$$
R_{u, v, w}=\left\{x \in A_{w} \mid \exists_{u^{\prime} \in A_{u} \wedge v^{\prime} \in A_{v}}\left\{u^{\prime}, v^{\prime}, x\right\} \in \mathcal{E}(\mathcal{B}[\mathcal{T}])\right\} .
$$

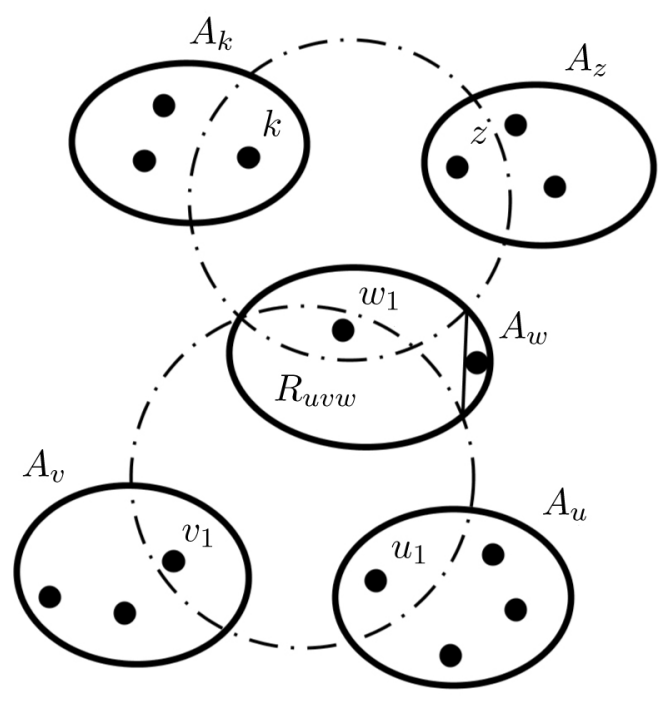

Figure 4. Clusters $A_{k}, A_{z}, A_{u}, A_{v}$ and $A_{w}$ (bold lines) with some hyperedges (broken lines) and the set $R_{u v w}$. 
Note that by (1)

$$
\left|R_{u, v, w}\right|\left|A_{u}\right|\left|A_{v}\right| \geq e\left(R_{u, v, w}, A_{u}, A_{v}\right)=e\left(A_{u}, A_{v}, A_{w}\right) \geq \gamma_{u v w}\left|A_{u}\right|\left|A_{v}\right|\left|A_{w}\right| .
$$

Hence $\left|R_{u, v, w}\right| \geq \gamma_{u v w}\left|A_{w}\right|$.

Now we give the lower bound for the number of hyperedges incident to $R_{u, v, w}$. Let $k, z \in V(\mathcal{T})$ be such that $\{k, z, w\} \in \mathcal{E}(\mathcal{T})$. By the inclusionexclusion formula we count a lower bound for the number of hyperedges between $R_{u, v, w}, A_{k}$ and $A_{z}$ as follows

$$
\begin{aligned}
e\left(R_{u, v, w},\right. & \left.A_{k}, A_{z}\right) \geq e\left(A_{w}, A_{k}, A_{z}\right)-\left(\left|A_{w}\right|-\left|R_{u, v, w}\right|\right) \cdot\left|A_{k}\right| \cdot\left|A_{z}\right| \\
& \geq\left|R_{u, v, w}\right| \cdot\left|A_{k}\right| \cdot\left|A_{z}\right|+\left(\gamma_{w k z}-1\right) \cdot\left|A_{w}\right| \cdot\left|A_{k}\right| \cdot\left|A_{z}\right| \\
& \geq\left|R_{u, v, w}\right| \cdot\left|A_{k}\right| \cdot\left|A_{z}\right|+\frac{1}{\gamma_{u v w}}\left(\gamma_{w k z}-1\right) \cdot\left|R_{u, v, w}\right| \cdot\left|A_{k}\right| \cdot\left|A_{z}\right| \\
& =\left(1-\frac{r_{w k z}}{1-r_{u v w}}\right) \cdot\left|R_{u, v, w}\right| \cdot\left|A_{k}\right| \cdot\left|A_{z}\right| \\
& =\gamma_{w k z}^{\prime} \cdot\left|R_{u, v, w}\right| \cdot\left|A_{k}\right| \cdot\left|A_{z}\right| .
\end{aligned}
$$

Now, by deleting the vertex set $A_{u}, A_{v}$ and $A_{w} \backslash R_{u, v, w}$ from $\mathcal{B}[\mathcal{T}]$, we obtain a hypergraph which is a blow-up hypergraph of $\mathcal{T}^{\prime}$ with hyperedge densities ensuring the existence of the factor $\mathcal{T}^{\prime}$. Moreover, by the definition of $R_{u, v, w}$ the factor $\mathcal{T}^{\prime}$ can be extended to a factor $\mathcal{T}$.

$(\Rightarrow)$ Note that if $\gamma_{w k z}^{\prime}<0$, then $\gamma_{w k z}+\gamma_{u v w}<1$. So there exists a construction which does not induce the linear hyperpath $\mathcal{P}_{2}$ with the consecutive vertices $u, v, w, k, z$ and hyperedges $\{u, v, w\},\{w, k, z\}$, where $i \in A_{i}$ $(i \in\{u, v, w, k, z\})$ in this case. Therefore, if some $\gamma_{w k z}^{\prime}<0$, then there exists a construction for a blow-up hypergraph of the hypertree $\mathcal{T}$ without a factor $\mathcal{T}$.

Next assume that all the $\gamma_{e}^{\prime}$ are proper densities, but there is a construction of a blow-up hypergraph, say $\mathcal{B}^{\prime}\left[\mathcal{T}^{\prime}\right]$, with hyperedge densities at least $\gamma_{e}^{\prime}$, but which does not induce a factor $\mathcal{T}^{\prime}$. Thus we construct a blow-up hypergraph $\mathcal{B}[\mathcal{T}]$ of the hypertree $\mathcal{T}$ not inducing $\mathcal{T}$. Namely, set $A_{w}=\left\{w^{*}\right\} \cup A_{w}^{\prime}, A_{u}=\{u\}$ and $A_{v}=\{v\}$. We create hyperedges $u, v, w$ for all $w \in A_{w}^{\prime}$ but do not create $u, v, w^{*}$ without changing densities in $\mathcal{B}^{\prime}\left[\mathcal{T}^{\prime}\right]$ and with an appropriate density $\gamma_{u v w}$.

Example 2. Let us consider the 3-uniform linear hypertree $\mathcal{T}$ with 5 hyperedges and 11 vertices presented in Figure 5(1) with two different sets of parameters $\left\{r_{e}\right\}_{e \in \mathcal{E}(\mathcal{T})}$ (in round brackets parameters $r_{e}$ from the second set of hyperedge densities are given). In Table 1, two different sets of densities $\left\{\gamma_{e}\right\}_{e \in \mathcal{E}(\mathcal{T})}, \gamma_{e}=1-r_{e}$, and changes of parameters $r_{e}$ during the execution of Algorithm $\mathcal{T}$ are presented. We are interested in whether these sets of hyperedge densities ensure the existence of the hypertree $\mathcal{T}$ as a factor. 
(1)

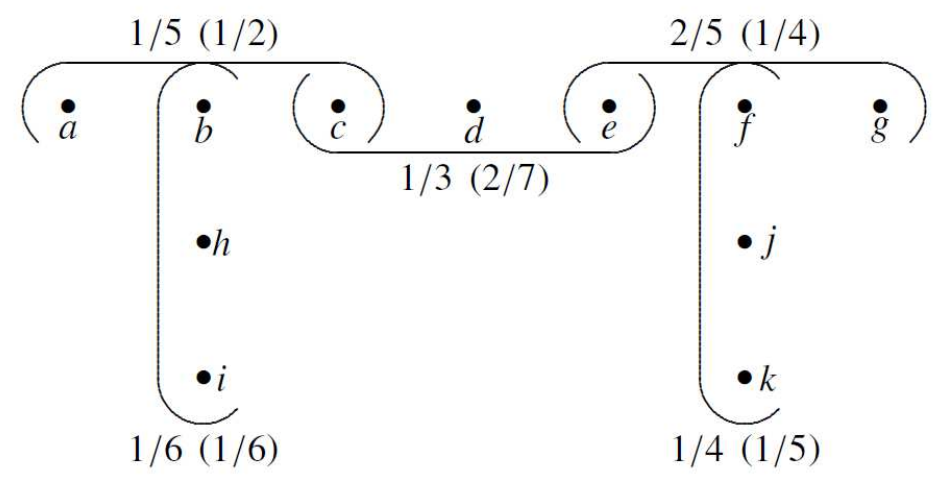

(2)

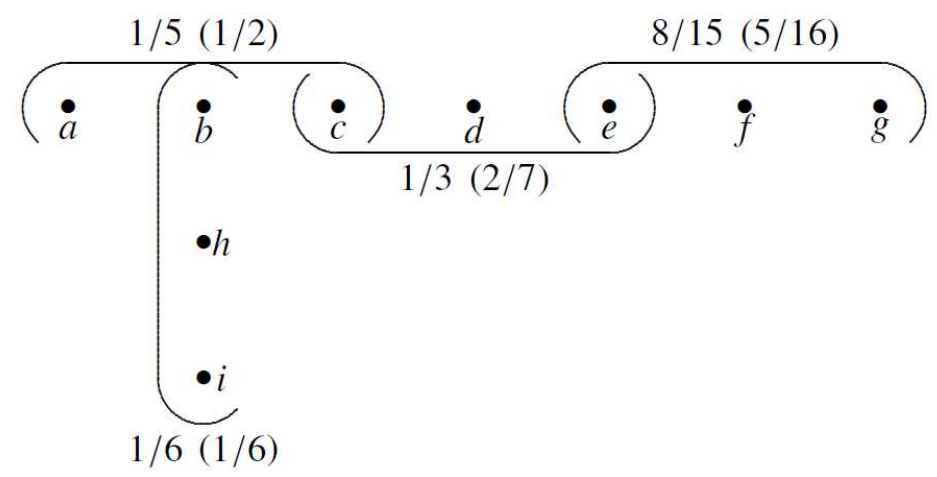

(3)

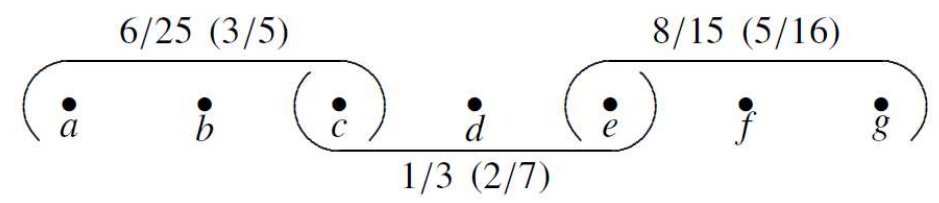

(4)

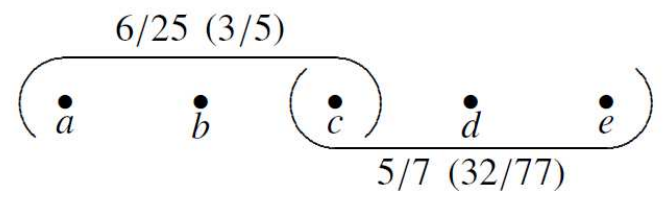

(5)

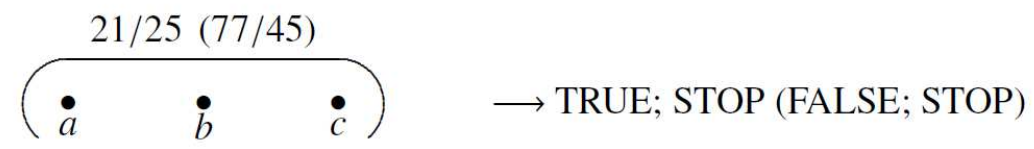

FiguRE 5. Executing Algorithm $\mathcal{T}$ for the hypertree $\mathcal{T}$ with two different sets of hyperedge densities. 


\begin{tabular}{|c||c|c|c|c|c|c||c|c|c|c|c|c|}
\hline \multicolumn{1}{|c||}{$e$} & \multicolumn{9}{c||}{ TRUE } & \multicolumn{6}{c|}{ FALSE } \\
\cline { 2 - 15 } & $\gamma_{e}$ & $(1) r_{e}$ & $(2) r_{e}$ & $(3) r_{e}$ & $(4) r_{e}$ & $(5) r_{e}$ & $\gamma_{e}$ & (1) $r_{e}$ & $(2) r_{e}$ & $(3) r_{e}$ & $(4) r_{e}$ & $(5) r_{e}$ \\
\hline \hline$a b c$ & $4 / 5$ & $1 / 5$ & $1 / 5$ & $6 / 25$ & $6 / 25$ & $21 / 25$ & $1 / 2$ & $1 / 2$ & $1 / 2$ & $3 / 5$ & $3 / 5$ & $77 / 45$ \\
\hline$c d e$ & $2 / 3$ & $1 / 3$ & $1 / 3$ & $1 / 3$ & $5 / 7$ & - & $5 / 7$ & $2 / 7$ & $2 / 7$ & $2 / 7$ & $32 / 77$ & - \\
\hline$e f g$ & $3 / 5$ & $2 / 5$ & $8 / 15$ & $8 / 15$ & - & - & $3 / 4$ & $1 / 4$ & $5 / 16$ & $5 / 16$ & - & - \\
\hline$b h i$ & $5 / 6$ & $1 / 6$ & $1 / 6$ & - & - & - & $5 / 6$ & $1 / 6$ & $1 / 6$ & - & - & - \\
\hline$f j k$ & $3 / 4$ & $1 / 4$ & - & - & - & - & $4 / 5$ & $1 / 5$ & - & - & - & - \\
\hline
\end{tabular}

TABLE 1. Changing parameters $r_{e}$ for hyperedges $e \in \mathcal{E}(\mathcal{T})$ during the execution of Algorithm $\mathcal{T}$ for two different sets of hyperedge densities $\gamma_{e}$ of the hypertree presented in Figure $5(1)$.

To solve this problem, we use Algorithm $\mathcal{T}$. For each hyperedge $e$ a parameter $r_{e}=1-\gamma_{e}$ is assigned as in Figure 5(1). Let us run Algorithm $\mathcal{T}$. All parameters satisfy the condition $0 \leq r_{e}<1$, so we cut the hyperedge $e_{f j k}$ and modify parameters $r_{e}$ by proper formulas presented in the algorithm. We repeat this procedure until we get a hypertree with a hyperedge $e^{*}$ for which parameter $r_{e^{*}} \geq 1$ or one-hyperedge hyperpath (see Figure $5(2)-(5)$ ). Notice that we get two different values at the end. First set of densities $\left\{\gamma_{e}\right\}$ ensures the existence of $\mathcal{T}$ as a factor and the second set $\left\{\gamma_{e}\right\}$ does not ensure.

We conclude with the following relation between roots of the multivariate matching polynomial and the inhomogeneous density Turán problem for 3uniform linear hypertrees.

Theorem 3. Let $\mathcal{T}=(V, \mathcal{E})$ be a weighted 3-uniform linear hypertree. Let $\gamma_{e}=1-t_{e}$ be densities assigned to each hyperedge $e \in \mathcal{E}(\mathcal{T})$, where $r_{e} \in$ $[0,1)$. Assume that after running Algorithm $\mathcal{T}$, we get a one-hyperedge hyperpath $\mathcal{P}_{1}$ with hyperedge weight equal to 0 . Then $t$ is a root of the multivariate matching polynomial $F_{\mathcal{T}}\left(\underline{r_{e}}, s\right)$ of the hypertree $\mathcal{T}$.

Proof. Let $\mathcal{T}=(V, \mathcal{E})$ be a weighted 3-uniform linear hypertree with $n$ hyperedges. To prove this theorem, we use induction on the number of hyperedges of the hypertree $\mathcal{T}$. If this hypergraph consists of 3 vertices (i.e., $\mathcal{T}$ is isomorphic to $\mathcal{P}_{1}$ ), then $F_{\mathcal{T}}\left(\underline{r_{e}}, t\right)=1-t r_{e}$ and the condition $F_{\mathcal{T}}\left(\underline{r_{e}}, t\right)=0$ means that $t$ is a root of this multivariate matching polynomial of the hypertree $\mathcal{T}$.

Assume that the statement is true for each hypertree on at most $n-1$ hyperedges. Let $\mathcal{T}$ be a hypertree with $n$ hyperedges and assume that we execute Algorithm $\mathcal{T}$ for a hyperedge $e=\{u, v, w\}$, shortly $u v w$, in the first step, where vertices $u, v$ are two leaves in $\mathcal{T}$. Let $\mathcal{T}^{\prime}=\mathcal{T}-\{u, v\}$ be a hypertee obtained from the hypertree $\mathcal{T}$ by deleting $u$ and $v$ and the hyperedge $\{u, v, w\}$. Densities on hyperedges in hypertree $\mathcal{T}^{\prime}$ are modified by formulas presented in Algorithm $\mathcal{T}$. By executing Algorithm $\mathcal{T}$, we 
obtain a one-hyperedge hyperpath $\mathcal{P}_{1}$ with $F_{\mathcal{P}_{1}}\left(\underline{r_{e}^{\prime}}, t\right)=0$. By induction we get that $F_{\mathcal{T}^{\prime}}\left(\underline{r_{e}}{ }^{\prime}, t\right)=0$.

We can expand $F_{\mathcal{T}^{\prime}}$ according to whether a monomial contains $x_{w k z}$ (where $w k z \in \mathcal{E}\left(\mathcal{T}^{\prime}\right)$ ) or does not. Obviously, each monomial contains at most one of the variables $x_{w k z}$, where $w k z \in \mathcal{E}(\mathcal{T})$.

Thus

$$
F_{\mathcal{T}^{\prime}}\left(\underline{x_{e}}, s\right)=Q_{0}\left(\underline{x_{e}}, s\right)-\sum_{\{k, z, w\} \in \mathcal{E}\left(\mathcal{T}^{\prime}\right)} s x_{w k z} Q_{k z}\left(\underline{x_{e}}, s\right),
$$

where $Q_{0}\left(x_{e}, s\right)$ consists of these terms which do not contain $x_{w k z}$ and $-s x_{w k z} Q_{k z}\left(\underline{x}_{e}, s\right)$ consists of these terms which contain $x_{w k z}$ (i.e., these terms correspond to the matchings containing the hyperedge $w k z)$.

We observe that

$$
F_{\mathcal{T}}\left(\underline{x_{e}}, s\right)=\left(1-s x_{u v w}\right) Q_{0}\left(\underline{x_{e}}, s\right)-\sum_{\{k, z, w\} \in \mathcal{E}\left(\mathcal{T}^{\prime}\right)} s x_{w k z} Q_{k z}\left(\underline{x_{e}}, s\right) .
$$

Since

$$
0=F_{\mathcal{T}^{\prime}}\left(\underline{r_{e}^{\prime}}, t\right)=Q_{0}\left(\underline{r_{e}}, t\right)-\sum_{\{k, z, w\} \in \mathcal{E}\left(\mathcal{T}^{\prime}\right)} t \frac{r_{w k z}}{1-t r_{u v w}} Q_{k z}\left(\underline{r_{e}}, t\right),
$$

we have

$$
\begin{aligned}
0= & \left(1-t r_{u v w}\right) F_{\mathcal{T}^{\prime}}\left(\underline{r_{e}^{\prime}}, t\right)=\left(1-t r_{u v w}\right) Q_{0}\left(\underline{r_{e}}, t\right) \\
& -\sum_{\{k, z, w\} \in \mathcal{E}\left(\mathcal{T}^{\prime}\right)} t r_{w k z} Q_{k z}\left(\underline{r_{e}}, t\right)=F_{\mathcal{T}}\left(\underline{r_{e}}, t\right) .
\end{aligned}
$$

Hence $t$ is a root of $F_{\mathcal{T}}\left(\underline{r_{e}}, s\right)$.

3. Conclusion. In this paper we showed some results for the inhomogeneous density Turán problem for 3-uniform linear hypertrees. We presented Algorithm $\mathcal{T}$ for testing whether the 3 -uniform linear hypertree $\mathcal{T}$ with the given set of hyperedge densities $\left\{\gamma_{e}\right\}_{e \in \mathcal{E}(\mathcal{T})}$ is a transversal of a blow-up hypergraph $\mathcal{B}[\mathcal{T}]$. By this algorithm we have the answer whether the hyperedge densities ensure the existence of the transversal or do not ensure. Moreover, we gave the theorem to prove the correctness of Algorithm $\mathcal{T}$.

We concluded with the theorem which shows the relation between the roots of the multivariate matching polynomial and the inhomogeneous density Turán problem for 3-uniform linear hypertrees.

Open problem. In the future work we want to solve the inhomogeneous density Turán problem for 3-uniform linear hypergraphs with one linear hypercycle. 


\section{REFERENCES}

[1] Baber, R., Johnson, J. R., Talbot, J., The minimal density of triangles in tripartite graphs, LMS J. Comput. Math. 13 (2010), 388-413, http://dx.doi.org/10.1112/S1461157009000436

[2] Berge, C., Graphs and Hypergraphs, Elsevier, New York, 1973.

[3] Bielak, H., Powroźnik, K., An efficient algorithm for the density Turán problem of some unicyclic graphs, in: Proceedings of the 2014 FedCSIS, Annals of Computer Science and Information Systems 2 (2014), 479-486, http://dx.doi.org/10.15439/978-83-60810-58-3

[4] Bollobás, B., Extremal Graph Theory, Academic Press, London, 1978.

[5] Bondy, A., Shen, J., Thomassé, S., Thomassen, C., Density conditions for triangles in multipartite graphs, Combinatorica 26 (2) (2006), 121-131, http://dx.doi.org/10.1007/s00493-006-0009-y

[6] Brown, W. G., Erdös, P., Simonovits, M., Extremal problems for directed graphs, J. Combin. Theory B 15 (1) (1973), 77-93, http://dx.doi.org/10.1016/0095-8956(73)90034-8.

[7] Csikvári, P., Nagy, Z. L., The density Turán problem, Combin. Probab. Comput. 21 (2012), 531-553, http://dx.doi.org/10.1017/S0963548312000016

[8] Füredi, Z., Turán type problems, in: (A. D. Keedwell, ed.) Survey in Combinatorics, 1991, Cambridge Univ. Press, Cambridge, 1991, 253-300, http://dx.doi.org/10.1017/cbo9780511666216.010.

[9] Godsil, C. D., Royle, G., Algebraic Graph Theory, Springer-Verlag, New York, 2001, http://dx.doi.org/10.1007/978-1-4613-0163-9.

[10] Jin, G., Complete subgraphs of r-partite graphs, Combin. Probab. Comput. 1 (1992), 241-250, http://dx.doi.org/10.1017/s0963548300000274

[11] Nagy, Z. L., A multipartite version of the Turán problem - density conditions and eigenvalues, Electron. J. Combin. 18 (1) (2011), Paper 46, 15 pp.

[12] Turán, P., On an extremal problem in graph theory, Mat. Fiz. Lapok 48 (1941), 436-452.

[13] Yuster, R., Independent transversal in r-partite graphs, Discrete Math. 176 (1997), 255-261, http://dx.doi.org/10.1016/s0012-365x(96)00300-7

Halina Bielak

Institute of Mathematics

Maria Curie-Skłodowska University

pl. M. Curie-Skłodowskiej 1

20-031 Lublin

Poland

e-mail: hbiel@hektor . umcs. lublin.pl
Kamil Powroźnik

Institute of Mathematics

Maria Curie-Skłodowska University

pl. M. Curie-Skłodowskiej 1

20-031 Lublin

Poland

e-mail: kamil . pawel . powroznik@gmail.com

Received March 20, 2018 